\title{
Fairness Improvement for Fixed Coordinated Beamforming Scheme in HetNets
}

\author{
Sirada Samomob ${ }^{1}$, Peerapong Uthansakul ${ }^{1}$, Monthippa Uthansakul ${ }^{1+}$ and Thossaporn Chanpuek ${ }^{2}$ \\ ${ }^{1}$ School of Telecommunication Engineering, Suranaree University of Technology, NakhonRatchasima, \\ Thailand 30000 \\ ${ }^{2}$ Faculty of Industrial Technology, Nakhonratchasima Rajabhat University, Nakhonratchasima, Thailand,
} 30000

\begin{abstract}
Coordinated beamforming has been considered as one famous method to tackle the problem of inter-cell interference for NetNets. So far, users occupying macrocell have higher signal-to-interference plus noise ratio comparing to the picocell users. This paper proposes a weighting scheme for coordinated beamforming in HetNets to give a fairness to both macrocell and picocell users.
\end{abstract}

Keywords: coordinated beamforming, HetNets, fairness.

\section{Introduction}

So far, the number of users for mobile communications has exponentially grown as the demand for realtime chatting, viewing multimedia and transferring big data has increased year by year. As a result, a large cell in cellular systems cannot provide a best satisfaction to all users. Then, there have been the installation of small cells inside a large cell for some areas having problem of weak signal or user congestion. The networks having both large and small cells inside are called Hetero-geneous Networks (HetNets). However, these networks are facing the problem of Inter-Cell Interference (ICI) as macrocell base station employs the same frequency as picocell base station does. According to this problem, users are experiencing a low Signal-to-Interference plus Noise (SINR).

Coordinated beamforming technology employing an antenna array cooperating with signal processing units has been considered to be a solution to tackle the problem of ICI. The macro base station needs to know the position of users within its own cell as well as the position of users in picocell. This is because the macro base station can perform beamforming avoiding ICI from picocell, vice versa for the picocell.

From literatures, many researchers have paid attention to the problem of ICI [1-3]. The authors of [1] have investigated into coordinated beamforming which is capable of solving the problem of ICI for cluster HetNets. They have also utilized sounding reference signal to deal with the channel estimation. In addition, the works presented in [2] have proposed a technique for coordinated beamforming when dealing the ICI problem. In that work, the proper base station for individual users can be chosen according to average channel gain. The concept of coordinated beamforming has been also studied in [3] in order to tackle the ICI problem. In that work, the cooperation between base stations have been introduced to reduce the ICI problem. The key success for coordinated beamforming is weighting coefficients.

There are 2 groups of weighting schemes. For the first one [4-6], weighting coefficients can be real-time calculated using some efficient algorithms such as zero forcing. The authors of [4] have introduced zero forcing to coordinated beamforming. For that work, users constantly need to feedback their information to

\footnotetext{
Corresponding author. Tel.: +66848355589; fax: +6644224603

E-mail address: mtp@sut.ac.th
} 
base station. Also, we can the help of zero forcing for coordinated beamforming in HetNets to reduce downlink co-channel interference in [5]. In addition, the authors of [6] have introduced the cooperation between zero forcing and block diagonalization for Coordinated MultiPoint (CoMP) transmission to give rise in users' sum rate. However, the drawback of this first group is that it hardware realization is limited as the weighting coefficients have be constantly updated. Also, zero forcing works well only when the communication is ideally noise free. For the second group, the weighting coefficients are predefined [7-9]. The authors of [7] have proposed the method to reduce the effect of downlink co-channel interference between macrocell and femtocell using uplink sounding reference signal to create the predefined weighting coefficients. Also in [8], (Signal to Leakage and Noise Ratio (SLNR) and Signal to Noise Ratio (SNR) have been utilized to find the best beamforming vector for the users. Moreover, [9] has presented how to calculate the predefined weighting coefficients at base station which can be applied to Coordinated Multi-Point Joint Processing (CoMP-JP). The advantage of this group is that the calculated weighting coefficients are practical. However, from literatures, users from other base station have not been taken into account for this type of calculation.

From the motivation mentioned earlier, this paper adopts the HetNet system when weighting coefficients are predefined. Also, the position of users in other cell coverage has to be taken into account when performing ICI cancellation. In addition, the fairness of users' SINR for both macrocell and picocell has to be considered.

\section{System Model}

From Fig.1, HetNets consist of 1 macrocell having 4 picocells staying inside its coverage area. Please note that all cells employ the same frequency and all the assumed scenarios are downlink. The received signal at the users of macrocell can be express as

$$
y_{U_{M}}=\sqrt{P_{M}} H_{U_{M}}^{M} W_{U_{M}} x_{U_{M}}+\sum \sqrt{P_{p}} H_{U_{M}}^{p} W_{U_{p}} x_{U_{p}}+n_{U_{M}}
$$

when $M$ and $p$ stand for macro and pico symbol, respectively. Also, $U_{M}$ and $U_{p}$ represent macrocell and picocell users, respectively.

Also, the received signal at the users of picocell can be express as

$$
y_{U_{p}}=\sqrt{P_{M}} H_{U_{p}}^{p} W_{U_{p}} x_{U_{p}}+\sqrt{P_{M}} H_{U_{p}}^{M} W_{U_{M}} x_{U_{M}}+\sum_{p^{\prime} \neq p} \sqrt{P_{p}} H_{U_{p}}^{p^{\prime}} W_{U_{p}^{\prime}} x_{U_{p}^{\prime}}+n_{U_{p}}
$$

When $H_{U_{M}}^{M}$ and $H_{U_{p}}^{M}$ are downlink channel matrix from base station to its own users and to picocell. Also, $H_{U_{M}}^{p}, H_{U_{M}}^{p}$ and $H_{U_{p}}^{p^{p}}$ are downlink channel matrix from picocell base station to its own users, to macrocell's users and to other picocell's users. The weighting coefficients $W$ is a predefined beamforming vector and $n$ stands for additive white Gaussian noise. The SNR for macrocell and picocell users can be express as: $\rho_{M}=P_{M} / \sigma_{n}^{2}, \rho_{p}=P_{p} / \sigma_{n}^{2}$ where $\sigma_{n}^{2}$ is noise power.

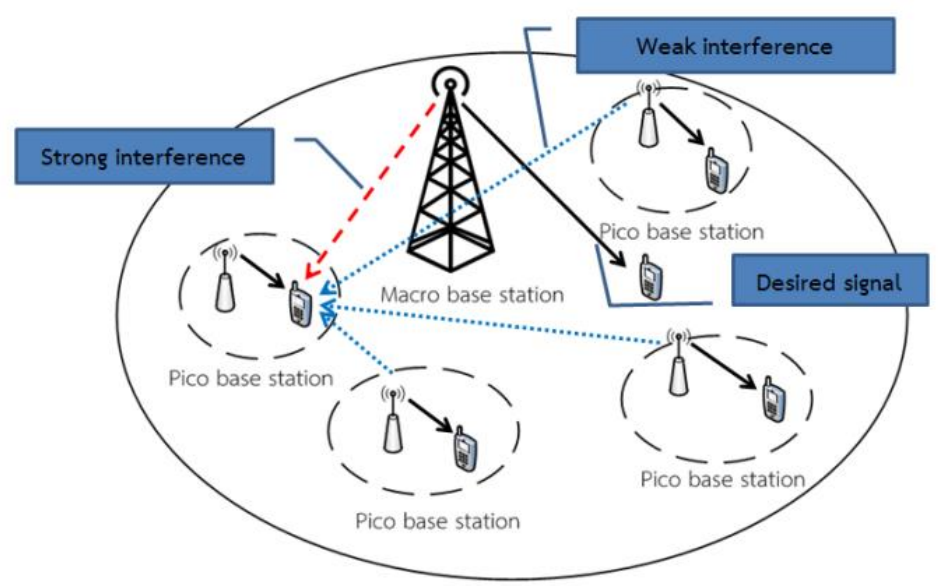

Fig. 1: HetNet model. 


\section{Proposed Scheme on Coordinated Beamforming}

The beamforming scheme proposed in this paper is a selection of weighting coefficients which give the maximum SINR to users as follows.

$$
W=\arg \max |\operatorname{SINR}|^{2}
$$

The SINR for users staying in macrocell can be expressed as

$$
\operatorname{SINR}_{U_{M}}=\frac{\rho_{M}\left|H_{U_{M}}^{M} W_{U_{M}}\right|^{2}}{\sum \rho_{p}\left|H_{U_{M}}^{p} W_{U_{p}}\right|^{2}+1}
$$

The SINR for users staying in picocell can be expressed as

$$
\operatorname{SINR}_{U_{p}}=\frac{\rho_{p}\left|H_{U_{p}}^{p} W_{U_{p}}\right|^{2}}{\rho_{M}\left|H_{U_{p}}^{M} W_{U_{M}}\right|^{2}+\sum_{p^{\prime \neq p}} \rho_{p}\left|H_{U_{p}}^{p^{\prime}} W_{U_{p}}\right|^{2}+1}
$$

The above expressions can provide the maximum to users for both macrocell and picocell users. However, users staying in macrocell always get higher SINR comparing with the users in picocell. This is not fair for users in picocell. Furthermore, this paper proposes a threshold to adjust the SINR for users in macrocell so we can have more degree of freedom to give rise in SINR value for picocell users. The following thresholds can give a fairness to users for both coverage areas.

$$
\operatorname{SINR}_{U_{M}}<16 d B, 10 d B \text { or } 5 d B
$$

Please note that those values can be referred to [10]. According to those thresholds, we can choose a proper weighting coefficient which give rise to SINR value at picocell users. However, those thresholds can be changed up to the satisfaction of network providers. The simulation results with and without setting the mentioned threshold will be discussed in next section.

\section{Simulation Results and Discussions}

This section shows some computer simulation of SINR for users at macrocell and picocell with and without giving the proposed thresholds as mentioned in last section. In the simulation, 1 macrocell and 4 picocells are assumed. Fig. 2 shows SINR for users staying in macrocell (MUE) and picocells (PUE1, PUE2, PUE3 and PUE4). As expected, macrocell user gets a higher SINR. Furthermore, picocell users get very low SINR in some points. This can cause a user dropped-call.



Fig.2: SINR for users in macrocell and picocell without setting threshold. 
Figs. 3 a) b) and c) show SINR values at users in macrocell and picocell when giving thresholds as the maximum SINR at macrocell user is $16 \mathrm{~dB}, 10 \mathrm{~dB}$ and $5 \mathrm{~dB}$, respectively, as mentioned in last section. As we can see, overall SINR values at picocell users increase. This can decrease a dropped-call rate to picocell users. The reason behind this success is that we can choose some appropriate weighting coefficients which limit the SINR at macrocell users while allowing higher SINR at picocell users. However, level of SINR at macrocell users drops comparing the ones without giving threshold. As we can see, the lower threshold we set, the higher SINR at picocell users we can get. However, the final SINR may not be the best value for macrocell users but it can give fairness to picocell users. This is a tradeoff that network providers have to concern.

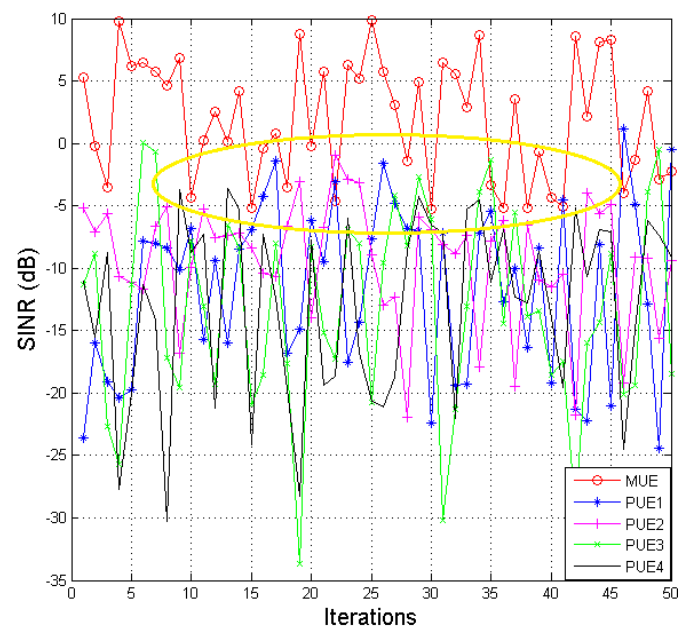

a)

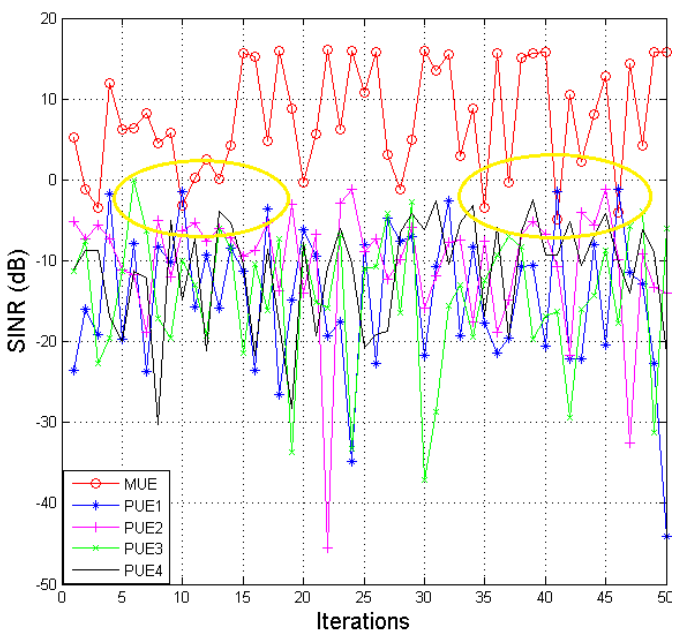

b)

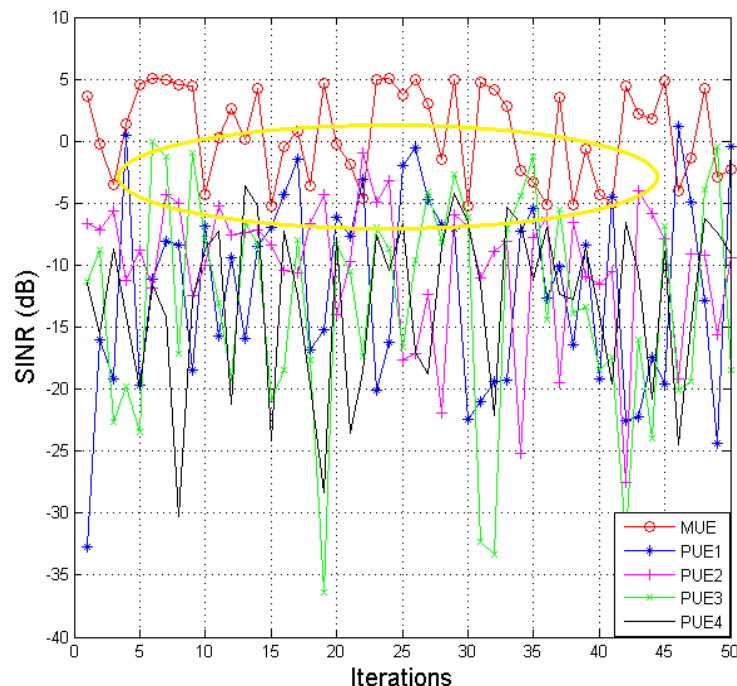

c)

Fig.3: SINR for users in macrocell and picocell when setting threshold as SINR of macrocell users a) $<16 \mathrm{~dB}$ b) $<10 \mathrm{~dB}$ and $\mathrm{c})<5 \mathrm{~dB}$.

\section{Conclusion}

This paper has investigated into the ICI problem in HetNets consisting of macrocell and picocell users, which can be solved using coordinated beamforming. So far, macrocell users always get higher SINR. So, a threshold has been introduced to give fairness to picocell users. This threshold allows us to choose some proper weighting coefficients which give rise to SINR at picocell users.

\section{References}

[1] S. Nagaraj, F. Hsieh, D. Pengoria, M. R. Raghavendra, M. Schamberger and M. Honig, "Coordinated beamforming in clustered HetNets: system design and performance evaluation", IEEE Wireless Communications and Networking Conference Workshops, pp.70-75, 6-9 April 2014. 
[2] O. Oguejiofor and L. Zhang, "Heuristic coordinated beamforming for heterogeneous cellular network", IEEE 83rd Vehicular Technology Conference (VTC Spring), pp. 1-5, 15-18 May 2016.

[3] J. Belschner,V. Rakocevic and J. Habermann, "The effect of out of cluster interference on coordinated beamforming in LTE-A HetNets", Communication Workshop (ICCW), pp. 203-209, 8-12 June 2015.

[4] L. Qiang, Y. Yang, F. Shu, “Zero-Forcing Beamforming with Limited Feedback in Coordinated Multi-point Transmission", Wireless Communications Networking and Mobile Computing (WiCOM), 23-25 September 2010.

[5] Y. Li, J. Li, L. Zhang and M. Peng, "Adaptive heterogeneous coordinated algorithm in LTE-advanced systems", IEEE Wireless Communications and Networking Conference (WCNC), pp.1893-1897, 7-10 April 2013.

[6] Md. H. A. Khan and M. H. Lee, "Zero-forcing beamforming with block diagonalization scheme for coordinated multi-point transmission", Communications (APCC), pp.152-156, 15-17 Oct. 2012.

[7] J. Kim, Y. Han and S. H. Lee, "An efficient downlink coordinated beamforming for heterogeneous networks", IEEE Vehicular Technology Conference (VTC Fall), pp.1-5, 2-5 Sept. 2013.

[8] Z. Huang, B. Li and M. Li, "Coordinated Beamforming of CoMP with limited feedback", Network Computing and Information Security (NCIS), pp. 138-141, 14-15 May 2011.

[9] P. Baracca, F. Boccadi, V. Braun and A. Tulino, "Base station selection and per-cell codebook optimization for CoMP with joint processing”, Personal Indoor and Mobile Radio Communications (PIMRC), pp.2329-2334, 9-12 Sept. 2012.

[10] Floatway systems, "LTE-RF drivetest and coverage analysis," http://www.slideshare.net/RayKhastur/lte-drivetestand-coverage-analysis. 